\title{
Beyond Ispagula - exploring alternative antifreeze infused laxative products as ultrasound medium for employment in regional anaesthetic training phantoms
}

\author{
Birk B. 1, Willers J.1, Uncles D. 1, Colucci G.2, Dearing J.1, Bisht L. 1
}

${ }^{1}$ Worthing Hospital, Western Sussex Hospitals NHS Foundation Trust, Dept of Anaesthesiology \& Intensive Care, Worthing, United Kingdom, 2Worthing Hospital, Western Sussex Hospitals NHS Foundation Trust, Dept of Surgery, Worthing, United Kingdom

\section{Background and Goal}

Previously, over a period of three years, we have developed and refined ADAMgel (Aqueous Dietary fibre Antifreeze Mix gel). This material is used as a tissue analogue for ultrasound based procedural training. It is produced by combining Ispagula (psyllium) husk, a commonly used dietary fibre laxative and food thickener mono ethylene glycol (MEG), a standard antifreeze, and water, then heating it. This resulting material satisfies the criteria for the ideal ultrasound medium $^{1}$ (IUM). Nevertheless we have continued to seek to improve the characteristics of this material and sought to establish whether other combinations of dietary fibre based laxative/food thickener ADAMgels have similar potential as IUMs.

\section{Materials and Methods}

We identified Gellan Gum (Fig.1) as being a practical alternative to psyllium in that it demonstrates sufficient viscoelasticity to support ultrasound probe pressure. Accordingly concentrations of $2,4,6$ and $8 \%$ were prepared with water in the ratio MEG 9/1 and tested against the criteria for IUM.

\section{Results and Discussion}

Gellan based ADAMgel performed favourably against the criteria for IUM. It reproduced high fidelity haptic human tissue simulation. Targets were clearly visible up to $10 \mathrm{~cm}$ and stayed fixed in position with clearly identifiable needle -target contact. This medium can be cast, moulded, cold-pressed or assembled in layers to create phantoms of varying complexity. It is non-perishable and has shown no deterioration after 6 months' storage at room temperature.

It can be prepared using basic equipment at $€ 3 / \mathrm{kg}$. Most significantly, it does not sustain needle insertion damage at gel concentrations < $8 \%$.(Fig. 2a-c). This is of particular relevance to phantom models that are likely to be subjected to multiple reuse.

\section{Conclusion}

Gellan based ADAMgel appears to be a useful medium for the basis of construction of regional anaesthesia training phantoms.

\section{References:}

Simulators for training in ultrasound guided procedures. 1. S. Sultan, G. Shorten, G Iohom Medical Ultrasonography 2013; 5,(2): 125-131
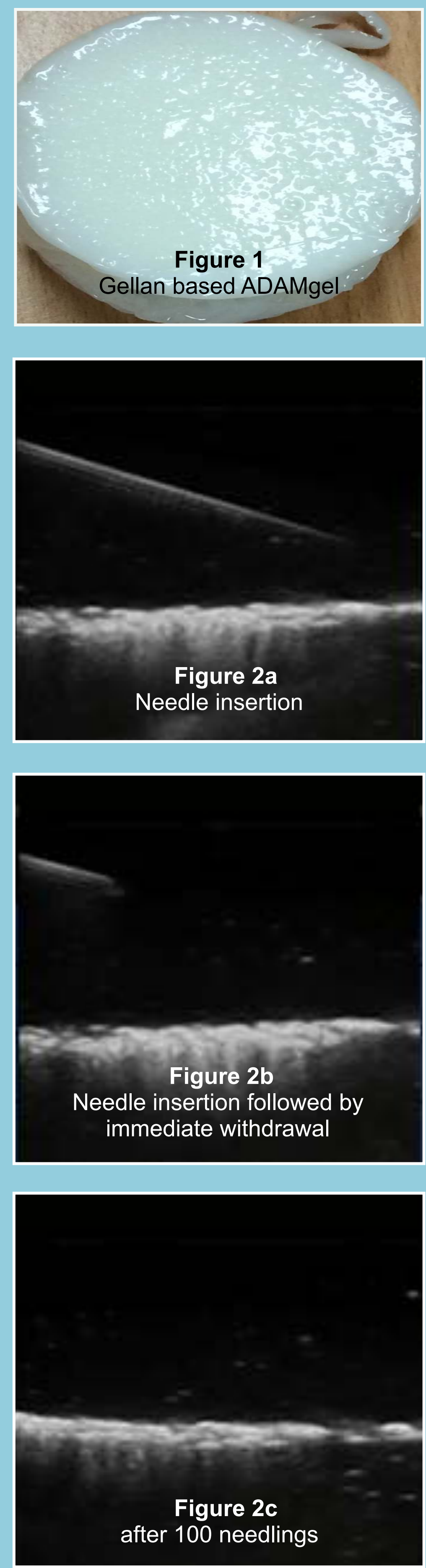